\title{
Controversies in revascularisation for stable coronary artery disease
}

\author{
Authors: Alexandra N Nowbar, ${ }^{\mathrm{A}}$ Christopher Rajkumar, ${ }^{\mathrm{A}}$ Rasha K Al-Lamee ${ }^{\mathrm{B}}$ and Darrel P Francis ${ }^{\mathrm{C}}$
}

\begin{abstract}
Recent randomised controlled trials, such as ISCHEMIA and ORBITA, have overturned most of what we were taught in medical school about hospital procedures considered necessary for patients with stable coronary artery disease. In this article, we discuss what these trials mean for physicians and patients considering revascularisation procedures with the hope of reducing the risk of death or alleviating angina.
\end{abstract}

KEYWORDS: stable angina, percutaneous coronary intervention, coronary artery disease, left main coronary disease, ischaemia

DOI: 10.7861/clinmed.2020-0922

\section{Coronary artery disease}

Coronary artery disease is the leading cause of death in the UK and worldwide. It can present acutely or as a chronic stable condition.

The key acute presentations of coronary artery disease are as myocardial infarction (MI) or unstable angina. In MI and some types of unstable angina, we know that urgent coronary revascularisation with percutaneous coronary intervention (PCI) or, in selected cases, coronary artery bypass grafting (CABG) reduces mortality. ${ }^{2}$ Patients are rightly advised to contact the emergency services directly rather than go through their GP so that they can go to a hospital by ambulance and have prompt life-saving treatment. This treatment regime includes several medications and revascularisation procedures proven by randomised trials to reduce mortality and further MI. The management of acute presentations of coronary artery disease is typically led by a cardiologist.

The chronic presentation of coronary artery disease is increasingly recognised to be a completely different clinical entity. Generally, these patients present to physicians in outpatient practice. It is understandable that GPs and patients might assume that the chronic presentation necessitates similar interventions to those required by patients presenting acutely, only without the same level of urgency. In this article, we will explore what recent

Authors: ${ }^{A}$ clinical research fellow, National Heart and Lung Institute, London, UK and Hammersmith Hospital, London, UK; ${ }^{B}$ Clinical senior lecturer, National Heart and Lung Institute, London, UK and Hammersmith Hospital, London, UK; ' professor of cardiology, National Heart and Lung Institute, London, UK and Hammersmith Hospital, London, UK trials have taught us about the dramatically different treatment required in acute versus chronic coronary artery disease.

\section{Each extra vessel of coronary disease doubles mortality}

Decades ago, the test for significant coronary artery disease was invasive coronary angiography with a view to referral for CABG because PCI was not available. Observational data, including the large CASS registry $(n=23,467)$, showed a progressive dramatic worsening of prognosis with increasing numbers of vessels with lesions the cardiologist considered significant. ${ }^{3}$ At 4 years, for example, the mortality was $4 \%$ for angiographically normal coronaries, $8 \%$ for one-vessel disease, $15 \%$ for two-vessel disease and $29 \%$ for three-vessel disease (Fig 1).

Outcomes for those who underwent CABG were better. Better outcomes in the surgery patients, together with the graded and indeed exponential increase in mortality risk as the number of diseased vessels increased, fostered a belief that stable coronary artery disease was potentially fatal without coronary revascularisation. ${ }^{4}$

However, associations between a phenomenon and mortality can easily arise due to confounding factors, even when the phenomenon does not cause death. For example, osteoarthritis is associated with cardiovascular mortality, and people receiving hip replacement for osteoarthritis have lower mortality than those who do not. However, this is not because osteoarthritis is fatal but simply

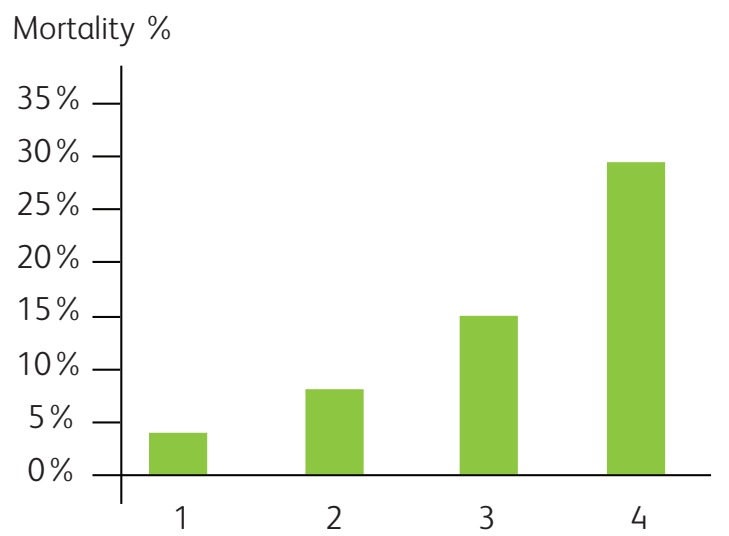

Fig 1. Number of diseased coronary arteries and mortality. Data from the CASS registry. ${ }^{3}$ 
that older age and obesity cause osteoarthritis and it is younger and healthier people who tend to undergo hip replacement.

\section{Ischaemia as a proxy for coronary disease}

While invasive angiography was accepted as the definitive method of quantifying coronary disease, this diagnostic procedure carried risks. A variety of non-invasive tests were developed that identified patients who were likely to have coronary disease on invasive testing. The earliest and most widely used was exercise ECG testing. Latterly, a variety of stress imaging tests promised higher sensitivity and/or specificity and, more interestingly, the ability to localise the likely region of coronary anatomy that was diseased.

This led to the concept of inducible ischaemia. The more abnormal the stress test, the worse the prognosis, both in terms of mortality and MI.

Fig 2 shows that three common non-invasive tests all predict subsequent events in a dose-dependent manner. It turned out that these tests are not unique, and every index of inducible ischaemia has the same property, namely the greater the level of ischaemia, the worse the outlook.

This reinforced the belief that the recurrent episodes of ischaemia that would likely arise in such patients were harmful. Ischaemia became a phenomenon sought out because it was thought to necessitate revascularisation. This search was led by cardiologists who specialised in imaging, be it by echocardiography, radionuclide scintigraphy or cardiovascular magnetic resonance.

\section{Invasive ischaemia as an immediate catheter laboratory proxy for non-invasive ischaemia}

Meanwhile, cardiologists performing diagnostic coronary angiography were becoming frustrated that having diagnosed coronary disease in the catheter lab, they would often have to send the patient home to have an outpatient non-invasive test to demonstrate ischaemia, if one had not been performed beforehand, which introduced delays.

Technology advanced so that pressure could be measured inside the coronary artery, allowing the pressure drop across a lesion to be measured as a surrogate for coronary blood flow. This measurement, known as fractional flow reserve (FFR), was now an objective measure of the physiological impact of anatomical stenoses on haemodynamics. Unsurprisingly, severe lesions tended to show a larger pressure drop than mild lesions. The advantage of these invasive indices of ischaemia was that they could be carried out immediately during angiography so that a decision could be made based on apparently objective criteria without delay.

Ironically, the evidence for there being a threshold in intracoronary pressures below which revascularisation was needed came from validation against non-invasive ischaemia markers, which in turn had originally been validated against coronary angiography. While quantification of a coronary stenosis by angiography was recognised as imperfect and vulnerable to observer bias, the intracoronary pressure measurements were considered sufficiently solid and convincing to be mandated by clinical guidelines. ${ }^{8}$ Indeed, in a further irony, non-invasive tests of ischaemia are now being validated against invasive FFR. ${ }^{9}$

\section{Unimpeachable evidence of therapeutic benefit from placebo-controlled trials}

In parallel with these advances in tests of coronary disease, an enormous body of randomised controlled trials of a

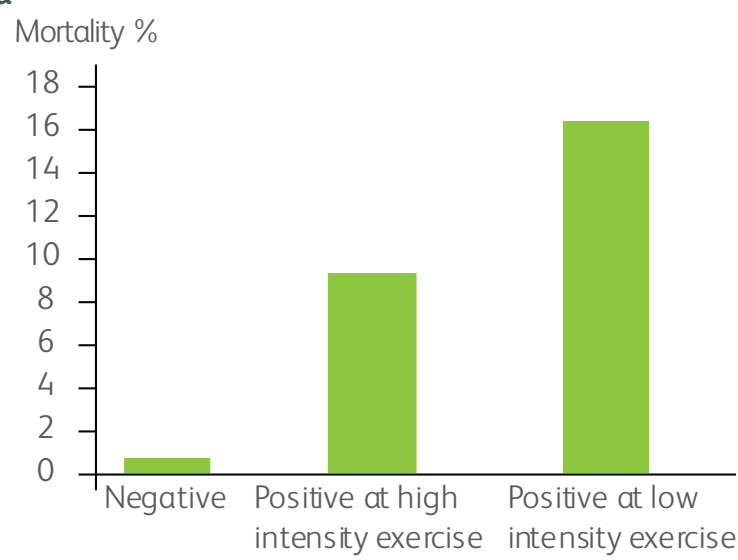

b

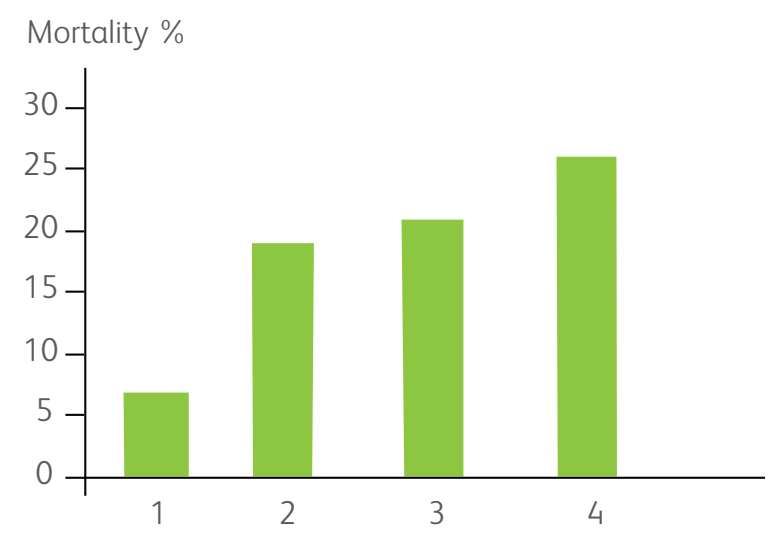

C

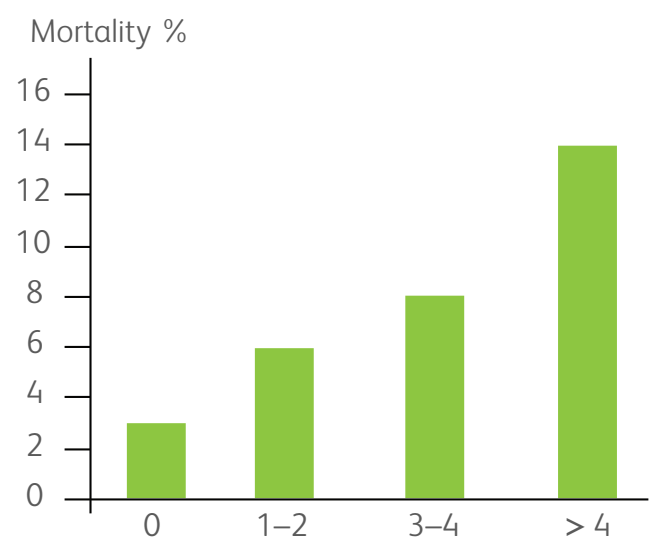

Fig 2. Relationship between three common non-invasive tests and mortality. (a) Exercise ECG and 5-year mortality, $n=429 .{ }^{5}$ (b) Stress echo and 8-year mortality, $n=3156 .{ }^{6}$ (c) Thallium SPECT and 2.5-year mortality, $n=340$.

pharmacotherapy, always placebo-controlled, established a variety of agents that were effective in achieving one or both of the major treatment goals in coronary disease, namely prevention of death or MI and reduction of angina. Although sometimes inappropriately grouped together as 'medical therapy', these agents are more rationally considered in terms of the therapeutic endpoints they seek to achieve. 
First, of the prognostically beneficial agents which have been repeatedly shown to reduce death or MI, the principal elements are statins and, for secondary prevention, antiplatelet drugs such as aspirin. Along with this come agents that reduce blood pressure such as ACE inhibitors.

The second group consists of those that alleviate angina, such as beta-blockers, calcium-channel blockers and nitrates. While there are a wide variety of such agents, the common theme is that, without exception, their effects have been demonstrated in placebo-controlled randomised trials.

\section{Testing whether ischaemia 'causes' rather than 'is associated with' mortality}

Observational data have suggested that the quantity of ischaemia or extent of coronary disease was powerfully associated with mortality (Fig 2) and that outcomes from patients who had undergone $\mathrm{CABG}$ or PCI were better than seemingly similar patients who had not. ${ }^{10}$

This was a persuasive story for the cardiology community. However, observational studies comparing recipients and non-recipients of a treatment are no substitute for randomised controlled clinical trials. While one can adjust for confounders such as age, the many different considerations that go into the decision to revascularise or not are much harder to measure and therefore control for. In standard clinical databases, they may not even be documented because they arise from powerful but difficult-toverbalise assessments such as the 'end of the bed test', which all physicians are familiar with.

The aptly named COURAGE trial tested the hypothesis that PCI would reduce mortality and MI rates. It randomised 2,287 patients with significant coronary disease and ischaemia to PCI or no PCI.11 To the surprise of many, although PCI was effective at reducing ischaemia, it had no effect on death and MI.

Some reacted with horror and argued that the trial results were incorrect. There were many criticisms, of which the most powerful was that physicians may have held back from randomising the patients who were most likely to benefit from PCI. In other words, once they saw a coronary angiogram with a very severe lesion, they might be too fearful to leave the patient without PCI. While this recruitment bias may have taken place, it is not likely to have contributed to the neutral result of COURAGE. We know this because, when stratified by the amount of ischaemia at baseline, there was no tendency for patients with more ischaemia to benefit more from PCI. ${ }^{12}$

The solution to a reluctance to randomise patients with a severe lesion is to randomise before the exact coronary anatomy is known. This was the approach taken in the subsequent and definitive $\$ 100$ million ISCHEMIA trial (International Study of Comparative Health Effectiveness with Medical and Invasive Approaches). ${ }^{13}$ It randomised 5,179 patients with moderate or severe ischaemia on stress testing to an initial invasive or conservative strategy before invasive coronary angiography based on a blinded core laboratory adjudicated CT coronary angiogram.

ISCHEMIA showed no difference in the primary endpoint of death from cardiovascular causes, MI or hospitalisation for unstable angina, heart failure, or resuscitated cardiac arrest.

Within the interventional cardiology community, this was again disappointing and has led to a search for a further fall-back position as we retreat from the grand expectation of a prognostic indication for revascularisation. Our final line of defence is that ISCHEMIA excluded certain groups of patients because leaving them without revascularisation was considered too risky (patients with left main stem disease on CT scan or poor left ventricular function) or too burdensome (for patients with severe symptoms). Randomised controlled data are still needed to understand if this is true.

\section{Patients still often assume that elective revascularisation is life-saving}

Patients often assume that they are making a choice between having elective PCI now versus having an infarct later, possibly requiring emergency intervention. ${ }^{14}$ In reality, almost all value in the prevention of coronary disease progression and subsequent MI and death comes from the medical treatment that has long been demonstrated to be very powerful.

The medications proven to reduce MI and death are so effective that it is difficult for any revascularisation procedure to show any additional benefit on this background. ${ }^{11}$ This is particularly true because any revascularisation procedure carries a small but nonnegligible risk of complications, including MI and death.

There may still be subgroups of coronary artery disease where elective revascularisation saves lives. For example, patients with left main stem disease were excluded from ISCHEMIA because omitting revascularisation in them was considered too risky. However, whether revascularisation for this sub-group is lifesaving remains unknown. We note that revascularisation for all patients was considered obviously life-saving until it was tested in randomised controlled trials.

When advising patients with stable coronary disease, physicians should take care to emphasise that it is the medications and not a revascularisation procedure that is key to saving their life.

\section{Testing whether revascularisation relieves symptoms}

Even if interventional cardiologists abandon life-saving claims for elective revascularisation, there is extensive evidence that it relieves anginal symptoms, and it is on this basis that guidelines can recommend it. ${ }^{15}$

Unfortunately, all of this evidence is unblinded. ${ }^{11,13,16-18}$ The reason unblinded evidence was accepted until recently was that there was an assumption of prognostic benefit, with symptom relief merely an incidental bonus. However, with the evaporation of the case for prognostic benefit, it has become more important to test symptom relief properly.

The Objective Randomised Blinded Investigation with optimal medical Therapy of Angioplasty in stable angina (ORBITA) trial randomised 200 patients to $\mathrm{PCI}$ or a placebo procedure on a background of multiple anti-anginal medications..$^{19}$ It showed no statistically significant improvement in exercise time beyond placebo. ${ }^{20}$ This does not mean that PCI had absolutely no effect on angina. It only means that any angina benefit was substantially smaller than previously believed. The belief was from the unblinded ACME trial, of plain balloon angioplasty without stenting, which showed $a+96$ second advantage of PCI over unblinded control with respect to improvements in exercise time. Since modern practice is to always insert a stent rather than only ballooning, because this is known to greatly reduce immediate vessel collapse and later re-stenosis, we might reasonably have expected the advantage of PCI to be much greater than 100 seconds. In reality, ORBITA showed a point estimate of just +16 seconds for PCI over placebo. In a non-prespecified analysis, however, ORBITA did show modest symptom relief with $\mathrm{PCI}$ in that one in five more patients were likely to be free from angina with PCI compared to placebo. Further 
analysis showed that the invasive ischaemia tests (which examine intracoronary pressures) were much less predictive of who would symptomatically benefit than expected. Guidelines indicate that a pressure ratio threshold exists below which revascularisation is indicated and above which it is not. ORBITA data showed not only no step in symptom response at this point, but in fact no detectable association between the pressure drop and the extent of benefit. ${ }^{21}$

However, ORBITA did show a surprisingly powerful predictive ability of the non-invasive ischaemia test: the more abnormal the baseline stress echo, the greater the placebo-controlled effect of PCI. ${ }^{20}$

Like any trial, ORBITA was imperfect, but it shared its imperfections with ACME, which showed a highly significant effect of PCI on exercise time. ${ }^{16}$ They did not differ in sample size, endpoint or statistical methodology. The main difference was that ORBITA was blinded with all patients undergoing either PCI or a placebo procedure and ACME was unblinded.

Just as our community did not like the results of COURAGE and demanded a rematch (ISCHEMIA), it did not like the results of ORBITA. A second trial is therefore now underway.

ORBITA-222 will advance on ORBITA in several ways. It will enrol patients with single or multi-vessel disease, not mandate guideline-directed anti-anginal therapy, and use symptoms as the primary endpoint rather than exercise treadmill testing.

Meanwhile, more attention is now being focused on the nature of angina symptoms. In recent decades, these have received relatively little attention in the face of a positive ischaemia test. However, experiments are now being performed under placebo control to test whether independent symptom verification during induced ischaemia can better select patients who will derive angina relief through PCI (ORBITA-STAR). ${ }^{23}$

\section{Left main stem disease}

A current controversy is how best to revascularise patients with left main stem disease. Disease in this location is considered so risky that none of the trials mentioned above randomised such patients.

Historically, the only way to revascularise left main disease was by CABG. However, not every patient is physically fit enough for CABG. To treat them, interventional cardiologists have developed methods for stenting the left main stem. Such methods are now a potential alternative for the generality of patients with left main stem disease.

The recent EXCEL trial of 1,905 patients ${ }^{24}$ addressed this question by randomising patients with left main stem disease to CABG or PCI. There was no difference in the primary outcome of death, stroke or MI at 5 years.

A previous trial, NOBLE, ${ }^{25}$ randomised 1,201 patients to CABG or PCI. It found CABG to be superior to PCI for the primary endpoint, a composite of all-cause mortality, non-procedural MI, repeat revascularisation and stroke.

A meta-analysis of five randomised controlled trials ${ }^{26}$ showed no difference in mortality between CABG and PCI, although some of these populations included both stable and unstable coronary artery disease patients. There was also no difference in MI, although procedural MI was more common after CABG and non-procedural MI more common after PCI. For stroke, there was again no difference except at the 12-month time point, which was reported by all five trials. At 12 months, the risk of stroke was lower in the PCI group (relative risk 0.38, 95\% confidence interval [CI] 0.19-0.77; $p=0.008$ ). Unplanned revascularisation was more common in the PCI group (risk ratio [RR] 1.73, 95\% confidence interval [CI] 1.49-2.02; $\mathrm{p}<0.001$ ). In a sensitivity analysis, the lack of difference in mortality between PCI and CABG was maintained when EXCEL was excluded.

In summary, for left main coronary artery disease, there is no appetite to conduct a randomised trial of revascularisation versus no revascularisation, because revascularisation is widely considered mandatory. The randomised trials of CABG versus PCI show similar outcomes between arms. The ultimate decision should be made by a heart team with cardiac surgeons and interventional cardiologists, taking into account surgical risk and patient preference. Risk scores can also predict individual cardiovascular risk to help guide the decision. ${ }^{27}$

\section{Summary}

Urgent revascularisation remains a cornerstone of the management of acute myocardial infarction, because of the proven survival benefits. However, for patients with stable coronary artery disease or stable angina, it should no longer be assumed that a mechanical solution to 'fix' a narrowed artery is obviously beneficial.

In angina, every licensed anti-anginal (of which there are many) has been proven to reduce angina by placebo-controlled trial. Placebo-controlled trials of PCI for angina are only now evolving. It is biologically plausible that PCI alleviates exertional angina, but this was not demonstrated in the only placebo-controlled trial completed so far and this remains an area of active study. Likewise it has been considered biologically plausible that revascularisation reduces mortality, but the ISCHEMIA trial and a meta-analysis of all such trials ${ }^{28}$ shows no such effect.

What would we do with a patient today with stable angina despite anti-anginal medication? We believe PCI may relieve their angina but have not been able to demonstrate this so far. Therefore we would enrol the patient into ORBITA-2.22 This is the rational approach in modern medicine when there is a treatment that has good theoretical grounds for relieving symptoms, but which has not yet been proven to do so, and for which a clinical trial exists.

In the modern era, the absolute priority in chronic stable coronary artery disease management is cardiovascular event prevention with medication and lifestyle measures. This is the responsibility of all physicians, and the prevention of cardiovascular disease remains a key goal of the NHS Long Term Plan.

\section{Key points}

> The primary aim in treatment of stable coronary artery disease is to prevent death and myocardial infarction (MI). Pharmacotherapy is excellent at this.

> The secondary aim is to alleviate symptoms. Pharmacotherapy is excellent at this too, because regulators have insisted on trials with placebo control as a rudimentary requirement for licensing.

$>$ Percutaneous coronary intervention (PCI) has been in use for $>40$ years for the relief of angina, but the first blinded placebo-controlled trial (ORBITA) showed an astonishingly weak effect, approximately one sixth of that seen in a comparable unblinded trial.

> Coronary revascularisation is widely perceived by patients and some physicians as necessary to prevent death in stable coronary artery disease. Cardiologists have also often tended to work on that assumption, though they have been more aware that randomised controlled trials have shown no mortality reduction.

> The ISCHEMIA trial has recently shown that in patients without significant left main stem disease, there is no mortality reduction from coronary revascularisation (be it by PCI or 
coronary artery bypass grafting), even though it recruited patients in whom strong mortality benefits were expected based on observational data.

$>$ We should not assume that treatments previously considered essential will show the large benefit that might be expected from observational data or from first principles.

$>$ Well controlled randomised trials require discipline, expense, and time, but are necessary to avoid vastly greater expenditure of resources over decades, and the missed opportunity to develop better interventions or patient selection.

\section{Conflicts of interest}

Rasha K Al-Lamee receives speaker's honoraria from Phillips Volcano and Menarini Pharmaceuticals. Alexandra N Nowbar is supported by the NIHR Academy. Christopher Rajkumar is supported by the MRC.

\section{References}

1 Nowbar AN, Gitto M, Howard JP et al. Mortality from ischemic heart disease. Circ Cardiovasc Qual Outcomes 2019;12:e005375.

2 Chacko L, P Howard J, Rajkumar C et al. Effects of percutaneous coronary intervention on death and myocardial infarction stratified by stable and unstable coronary artery disease: a meta-analysis of randomized controlled trials. Circ Cardiovasc Qual Outcomes 2020;13:e006363.

3 Emond M, Mock MB, Davis KB, Fisher LD, Holmes DR, Chaitman $B R$, et al. Long-term survival of medically treated patients in the Coronary Artery Surgery Study (CASS) Registry. Circulation 1994:90:2645-57.

4 Task Force on Myocardial Revascularization of the European Society of Cardiology (ESC) and the European Association for Cardio-Thoracic Surgery (EACTS), European Association for Percutaneous Cardiovascular Interventions (EAPCI) et al. Guidelines on myocardial revascularization. Eur Heart ] 2010;31:2501-55.

5 Severi S, Picano E, Michelassi C et al. Diagnostic and prognostic value of dipyridamole echocardiography in patients with suspected coronary artery disease. Comparison with exercise electrocardiography. Circulation 1994;89:1160-73.

6 Marwick TH, Case C, Sawada S et al. Prediction of mortality using dobutamine echocardiography. J Am Coll Cardiol 2001;37:754-60.

7 Snader CE, Marwick TH, Pashkow FJ et al. Importance of estimated functional capacity as a predictor of all-cause mortality among patients referred for exercise thallium single-photon emission computed tomography: report of 3,400 patients from a single center. J Am Coll Cardiol 1997;30:641-8.

8 Neumann F-J, Sousa-Uva M, Ahlsson A et al. 2018 ESC/EACTS Guidelines on myocardial revascularization. Eur Heart J 2019;40: 87-165.

9 Li Min, Zhou Tao, Yang Lin-feng et al. Diagnostic accuracy of myocardial magnetic resonance perfusion to diagnose ischemic stenosis with fractional flow reserve as reference. JACC CardiovasC Imaging 2014;7:1098-105.

10 Windecker S, Stortecky S, Stefanini GG et al. Revascularisation versus medical treatment in patients with stable coronary artery disease: network meta-analysis. BMJ 2014:348:g3859.

11 Boden WE, O'Rourke RA, Teo KK et al. Optimal medical therapy with or without PCI for stable coronary disease. N Engl J Med 2007:356:1503-16.

12 Shaw LJ, Weintraub WS, Maron DJ et al. Baseline stress myocardial perfusion imaging results and outcomes in patients with stable ischemic heart disease randomized to optimal medical therapy with or without percutaneous coronary intervention. Am Heart ] 2012;164:243-50.
13 Maron DJ, Hochman JS, Reynolds HR et al. Initial invasive or conservative strategy for stable coronary disease. N Engl J Med 2020;382:1395-407.

14 Rothberg MB, Scherer L, Kashef MA et al. The effect of information presentation on beliefs about the benefits of elective percutaneous coronary intervention. JAMA Intern Med 2014;174:1623-9.

15 National Institute of Health and Care Excellence. Stable angina: management. Clinical guideline [CG126]. NICE, 2011. Available from www.nice.org.uk/guidance/cg126.

16 Folland ED, Hartigan PM, Parisi AF. Percutaneous transluminal coronary angioplasty versus medical therapy for stable angina pectoris: outcomes for patients with double-vessel versus single-vessel coronary artery disease in a Veterans Affairs Cooperative randomized trial. J Am Coll Cardiol 1997;29:1505-11.

17 De Bruyne B, Pijls NHJ, Kalesan B et al. Fractional flow reserveguided PCI versus medical therapy in stable coronary disease. $N$ Engl J Med 2012;367:991-1001.

18 Coronary angioplasty versus medical therapy for angina: the second Randomised Intervention Treatment of Angina (RITA-2) trial. Lancet 1997;350:461-8.

19 Al-Lamee R, Thompson D, Dehbi H-M et al. Percutaneous coronary intervention in stable angina (ORBITA): a double-blind, randomised controlled trial. Lancet 2018;391:31-40.

20 Al-Lamee RK, Shun-Shin MJ, Howard JP et al. Dobutamine stress echocardiography ischemia as a predictor of the placebo-controlled efficacy of percutaneous coronary intervention in stable coronary artery disease: the stress echocardiography-stratified analysis of ORBITA. Circulation 2019 10;140:1971-80.

21 Al-Lamee R, Howard JP, Shun-Shin MJ et al. Fractional flow reserve and instantaneous wave-free ratio as predictors of the placebo-controlled response to percutaneous coronary intervention in stable single-vessel coronary artery disease. Circulation 2018;138:1780-92.

22 Imperial College London. A placebo-controlled trial of percutaneous coronary intervention for the relief of stable angina. https:// clinicaltrials.gov/ct2/show/NCT03742050.

23 Imperial College London. Symptomatic trial of angina assessment prior to revascularization. https://clinicaltrials.gov/ct2/show/ NCT04280575.

24 Stone GW, Kappetein AP, Sabik JF et al. Five-year outcomes after PCI or CABG for left main coronary disease. N Engl ] Med 2019;381:1820-30

25 Mäkikallio T, Holm NR, Lindsay M et al. Percutaneous coronary angioplasty versus coronary artery bypass grafting in treatment of unprotected left main stenosis (NOBLE): a prospective, randomised, open-label, non-inferiority trial. Lancet 2016;388:2743-52.

26 Ahmad Y, Howard JP, Arnold AD et al. Mortality after drug-eluting stents vs. coronary artery bypass grafting for left main coronary artery disease: a meta-analysis of randomized controlled trials. Eur Heart ] 2020;41:3228-35

27 Takahashi K, Serruys PW, Fuster V et al. Redevelopment and validation of the SYNTAX score II to individualise decision making between percutaneous and surgical revascularisation in patients with complex coronary artery disease: secondary analysis of the multicentre randomised controlled SYNTAXES trial with external cohort validation. Lancet 2020, in press (doi: 10.1016/S01406736(20)32114-0).

28 Bangalore S, Maron DJ, Stone GW, Hochman JS. Routine revascularization versus initial medical therapy for stable ischemic heart disease. Circulation 2020;142:841-57.

Address for correspondence: Dr Alexandra N Nowbar, B block South, 2nd floor, National Heart and Lung Institute Cardiovascular Science, Du Cane Road, London W12 0NN, UK. Email: alexandra.nowbar09@imperial.ac.uk Twitter: @AlexNowbar, @rajkumar_chris, @rallamee, @ProfDFrancis 